\title{
Natural occurrence of deoxynivalenol (DON) in wheat based noodles consumed in Malaysia.
}

\begin{abstract}
In this first study performed in noodle samples consumed in Malaysia for the presence of deoxynivalenol (DON), a total of 135 sample of noodles, comprised of instant noodle, yellow alkaline noodle and white salted noodle, were randomly collected from food stores and analyzed for DON using high performance liquid chromatography (HPLC) with a PDA (photodiode array) detector at $218 \mathrm{~nm}$. The objective of this study was to investigate the DON contamination levels in different types of wheat-based noodle consumed in Peninsular Malaysia. An acetonitrile:water (17:83 v/v) mixture was used as a mobile phase and clean-up was accomplished with a Mycosep 225 column. There was a high variation in the DON concentrations in all types of noodle group as well as between brands. Only one sample of instant and yellow alkaline noodle each were contaminated with DON, at concentrations of 1.003 and $1.243 \mathrm{ng} / \mathrm{g}$, respectively. The minimum detectable concentration for the DON was $0.627 \mathrm{ng} / \mathrm{g}$ in instant noodle. In the case of white salted noodle, none of the samples contained any detectable amount of DON. The results indicate a low occurrence of DON mycotoxins in commercial noodle products in Malaysia.
\end{abstract}

Keyword: Deoxynivalenol; Noodles; HPLC. 